\title{
On the Influence of Ship Motion Prediction Accuracy on Motion Planning and Control of Robotic Manipulators on Seaborne Platforms
}

\author{
Pål J. From ${ }^{1} \quad$ Jan T. Gravdahl ${ }^{1} \quad$ Pieter Abbeel $^{2}$ \\ ${ }^{1}$ Department of Engineering Cybernetics, Norwegian University of Science and Technology, Trondheim, Norway \\ ${ }^{2}$ Department of EECS, University of California, 253 Cory Hall, Berkeley, CA 94720-1770, USA \\ \{from,tommy.gravdahl\}@itk.ntnu.no pabbeel@cs.berkeley.edu
}

\begin{abstract}
Robotic manipulators on non-inertial platforms, such as ships, have to endure large inertial forces due to the non-inertial motion of the platform. When the non-inertial platform's motion is known, motion planning and control algorithms can eliminate these perturbations-in fact, in some situations the motion planning algorithms can even leverage the inertial forces to more cheaply move to a target point. However, for many non-inertial platforms, the motion is unknown.

In this paper we investigate how prediction errors and the choice of the prediction horizon affect the motion planning and control of robots mounted on a non-inertial base with a particular focus on seaborne platforms. We study the following three aspects: (i) We study prediction of ship motion and how prediction errors affect the motion planning and control of the manipulator. (ii) We evaluate the relationship between prediction accuracy and control. In particular, we study what prediction horizon length is useful for motion planning and control. We also consider how uncertainties in the ship motion predictions map to uncertainties in the future state of the robot and how to include the variance in the cost function to increase the optimal horizon length. (iii) Finally, we study a receding horizon approach, which re-solves the optimal control problem on-line over a horizon as determined to be meaningful from (ii). Several simulations are presented and, to our knowledge, for the first time experiments of ship-manipulator systems based on real ship motion data are presented.
\end{abstract}

\section{INTRODUCTION}

Ships and oil platforms are expected to become increasingly unmanned in the future and hence the need for autonomously operating robots for surveillance, maintenance, and operation will continue to increase over time [1], [2]. The demand for unmanned operation becomes even higher in harsh environments such as high sea state (Figure 1), when it can be dangerous for human operators to be exposed. High sea environments are not only dangerous for human operators, they also pose significant challenges for robotic control: Large inertial forces will influence the manipulator and, when not anticipated and accounted for, can make the operation inaccurate, extremely energy demanding, and sometimes even impossible due to torque limits. The inertial forces thus need to be taken into account in both the path planning and control of the robot.

In From et al. [3] the authors solve the problem of optimal motion planning for a robot mounted on a ship under the assumption the base motion is known for all times. The approach includes the ship motion in the trajectory planning problem and an optimal trajectory in terms of actuator torques is found. However, in most practical situations the

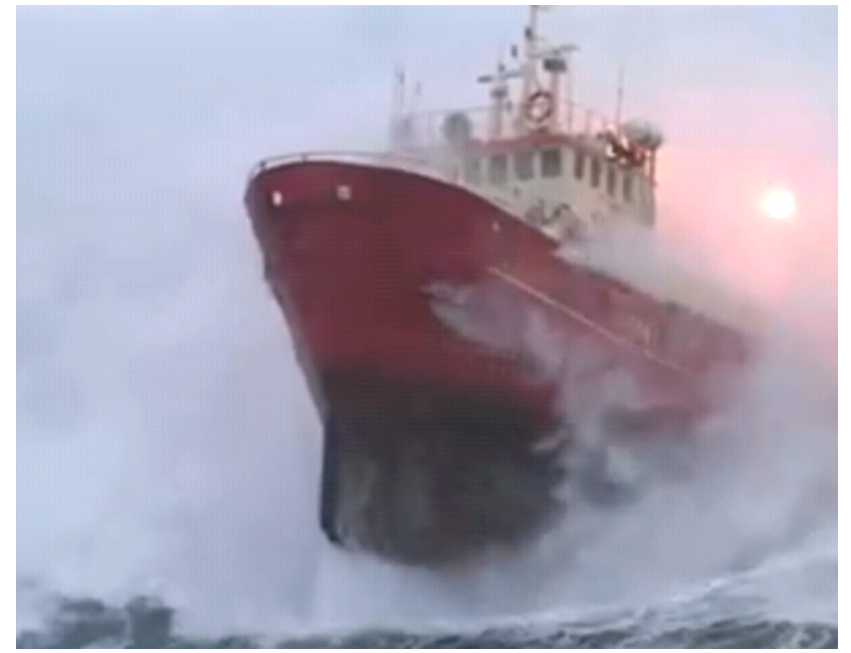

Fig. 1. A ship in high sea. The wave forces can result in very high accelerations in the ship motion.

forces acting on the ship due to the interaction with the waves and the wind are very complex and one cannot expect to know the base motion for all times.

The extent to which we can obtain accurate ship motion predictions thus directly determines how well we can compensate for or take advantage of the inertial forces. However, the wave-ship system is not deterministic, and accurately predicting the ship motion is a challenging problem. The prediction accuracy depends on the sea state, the ship, the ship and wave models available, and on the methods used to predict the ship motion.

The accuracy of the ship motion prediction not only directly determines how optimal a solution we can achieve, it also affects the computational requirements. In a receding horizon setting, where the optimal control inputs sequence is re-computed at regular intervals, the computational burden will increase for an inaccurate model: For an inaccurate model the initialization point, taken from the previous solution, is further away from the optimal solution. In addition to affecting the choice of horizon the modeling error can thus directly affect the frequency for which the optimal control or optimal trajectory can be recalculated.

A good understanding of the complete system-including the robot, the ship and waves, and the uncertainties-is essential in deciding on what measuring equipment and 
algorithms are required to obtain an optimal solution. For example, cameras could be used to obtain information about the size and the direction of the next wave, which can reduce the prediction errors and improve performance. The choice of equipment and algorithms will directly affect the horizon over which predictions are accurate and thus also directly influence control performance.

In this paper we investigate how well we can compensate for the inertial forces using measurements of the ship motion only. An important contribution of this paper is that we use real data, i.e., measurements taken from a full scale ship. Much of the literature on ship motion prediction use computer generated data for comparison, which leads to unrealistically small errors in the predictions.

Stochastic uncertainty is present in a wide variety of systems, ranging from mechanical systems and process control to finance. In general, receding horizon control is a well suited control scheme to deal with uncertainties, but most approaches do not use information about the probability distribution governing the uncertainty and they only assume that the uncertainty is bounded. Thus, the information about the probabilistic distribution is ignored and the worst-case representation of the disturbances or constraints often leads to a conservative solution.

In this paper we present a new motion planning algorithm that also minimizes the variance of the controlled state. First, the use of real ship motion measurements allows us to calculate the variance of the predictions of the forced state. We find that the variance is different for roll, pitch and yaw. Second, we illustrate how we can use the Extended Kalman filter to find how the variance in the forced state maps to the variance of the controlled state, i.e., how the uncertainties in the ship motion predictions map to uncertainties in the robot state. Finally we show how we can choose a robot trajectory for which the variance of the controlled state (the robot) is minimized, assuming the variance of the forced state is known. We find that augmenting the cost function to also include the variance allows us to choose a longer horizon than when the variance is not included.

\section{Related Research}

Research on several related topics can be found in the literature. Love et al. [1] addressed the impact of wave generated disturbances on the tracking control of a manipulator mounted on a ship based on the classical Lagrangian approach. They used repetitive learning control and this resulted in performance improvement for purely periodic motions, but they did not present a formal derivation of the dynamics equations. Kitarovic et al. [2] and Oh et al. [4] addressed the use of cable robots for loading and unloading cargo between two ships. In the Ampelmann project [5], a Stewart platform is mounted on a ship and is used to compensate for the motion of the ship by keeping the platform still with respect to the world frame. Lebans et al. [6] give a cursory description of a telerobotic shipboard handling system, and Kosuge et al. [7], [8] address the control of robots floating on the water utilizing vehicle restoring forces. Other related research areas are macro/micro manipulators [9], [10], underwater vehicle/manipulator systems [11] and

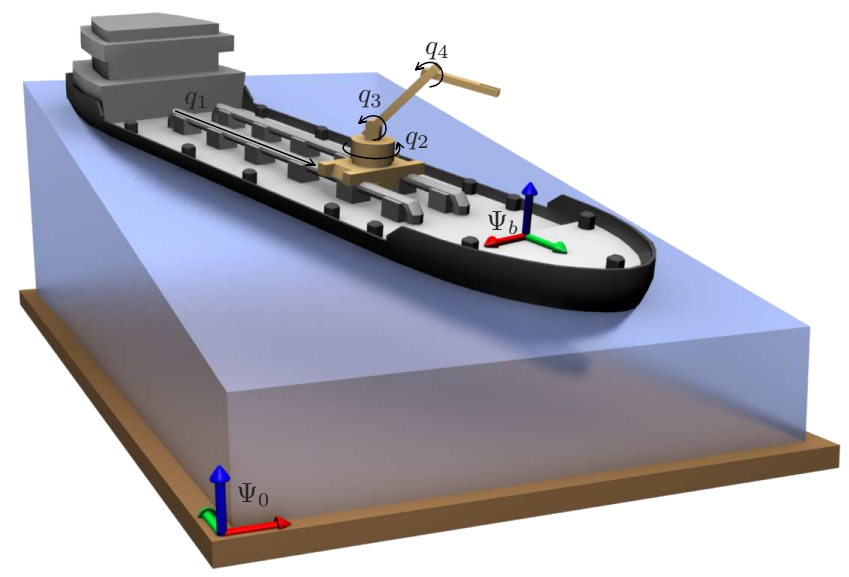

Fig. 2. Model setup for a four-link robot attached to a non-inertial base with coordinate frame $\Psi_{b}$. Frame $\Psi_{0}$ denotes the inertial reference frame.

spacecraft/manipulator systems [12]. Most previous work deals with robots mounted on a free-floating base. There is, however, an important difference between modeling a robot on a forced and a free-floating base. A forced base motion will add inertial forces to the dynamic equations that do not arise in the free-floating case, such as spacecraft/manipulator systems and manipulators on small AUVs.

There are some papers in the literature considering the prediction of ship motion. Yang et al. [13], [14] discuss the problem of landing a helicopter on a ship in high sea and predict the ship motion by fitting the ship model to the measured data using recursive least squares. Khan et al. [15] use artificial neural networks to solve the same problem. The Auto-Regressive fitting model is easy to implement but accurate predictions can only be obtained for a short horizon. If the principal frequencies are known the ship motion can be written as a superposition of $N$ sinusoidal waves with different frequencies. In Chung et al. [16] the sea excitation is extrapolated using this approach and the ship motion is predicted using the ship model.

\section{Ship-Manipulator Modeling}

In From et al. [3] the classical dynamics equations for a serial manipulator arm with 1-DoF joints were extended to include the forced 6-DoF motion of the base. For more details on how to derive the dynamics see From et al. [3] or Duindam et al. [17], [18]. We consider the setup of Figure 2 describing a general $n$-link robot manipulator arm attached to a moving base. We choose an inertial coordinate frame $\Psi_{0}$, a frame $\Psi_{b}$ rigidly attached to the moving base, and $n$ frames $\Psi_{i}$ (not shown) attached to each link $i$ at the center of mass. Finally, we choose a vector $q \in \mathbb{R}^{n}$ that describes the configuration of the $n$ joints. Using standard notation [19], we can describe the pose of each frame $\Psi_{i}$ relative to $\Psi_{0}$ as a homogeneous transformation matrix $g_{0 i} \in S E(3)$. This pose can also be described using the vector of joint coordinates $q$ as

$$
g_{0 i}=g_{0 b} g_{b i}(q) .
$$

The base pose $g_{0 b}$ and the joint positions $q$ thus fully determine the configuration of the robot. 
In a similar way, the spatial velocity of each link can be expressed using twists [19]:

$$
V_{0 i}^{0}=\left[\begin{array}{c}
v_{0 i}^{0} \\
\omega_{0 i}^{0}
\end{array}\right]=V_{0 b}^{0}+V_{b i}^{0}=\operatorname{Ad}_{g_{0 b}}\left(V_{0 b}^{b}+J_{i}(q) \dot{q}\right)
$$

where $v_{0 i}^{0}$ and $\omega_{0 i}^{0}$ are the linear and angular velocities, respectively, of link $i$ relative to the inertial frame, $J_{i}(q) \in$ $\mathbb{R}^{6 \times n}$ is the geometric Jacobian of link $i$ relative to $\Psi_{b}$, the adjoint is defined as $\operatorname{Ad}_{g}:=\left[\begin{array}{cc}R & \hat{p} R \\ 0 & R\end{array}\right] \in \mathbb{R}^{6 \times 6}$, and $\hat{p} \in \mathbb{R}^{3 \times 3}$ is the skew symmetric matrix such that $\hat{p} x=p \times x$ for all $p, x \in \mathbb{R}^{3}$. The velocity state is thus fully determined given the twist $V_{0 b}^{b}$ of the base and the joint velocities $\dot{q}$.

We can write the dynamic equations in block-form as

$$
\left[\begin{array}{cc}
M_{V V} & M_{q V}^{T} \\
M_{q V} & M_{q q}
\end{array}\right]\left[\begin{array}{c}
\dot{V}_{0 b}^{b} \\
\ddot{q}
\end{array}\right]+\left[\begin{array}{ll}
\bar{C}_{V V} & \bar{C}_{V q} \\
\bar{C}_{q V} & \bar{C}_{q q}
\end{array}\right]\left[\begin{array}{c}
V_{0 b}^{b} \\
\dot{q}
\end{array}\right]=\left[\begin{array}{c}
F_{b}^{b} \\
\tau
\end{array}\right]
$$

with $F_{b}^{b}$ the external wrench on the base link, expressed in coordinates $\Psi_{b}$ (such that it is collocated with the twist $V_{0 b}^{b}$ ).

We are interested in the effects that the pose $g_{0 b}$, the velocity $V_{0 b}^{b}$, and the acceleration $\dot{V}_{0 b}^{b}$ have on the manipulator dynamics. We can see this by rewriting the dynamics as

$$
M_{q q} \ddot{q}+\bar{C}_{q q} \dot{q}+\underbrace{M_{q V} \dot{V}_{0 b}^{b}+\bar{C}_{q V} V_{0 b}^{b}}_{\text {inertial forces }}=\tau .
$$

Finally, the way the gravitational forces translate into joint torques depends on the configuration of the base and need to be added to the right hand side of (4). The torque associated with link $i$ is given by

$$
\tau_{g}^{i}=J_{i}(q) \operatorname{Ad}_{g_{0 i}}^{\top}(Q) F_{g}^{i}(Q)
$$

Note that both $R_{0 i}$ and $\operatorname{Ad}_{g_{0 i}}$ depend on the base configuration with respect to the inertial frame. $F_{g}^{i}$ is given by

$$
F_{g}^{i}=\left[\begin{array}{c}
f_{g} \\
\hat{r}_{g}^{i} f_{g}
\end{array}\right]=-m_{i} g\left[\begin{array}{c}
R_{0 i} e_{z} \\
\hat{r}_{g}^{i} R_{0 i} e_{z}
\end{array}\right]
$$

where $e_{z}=\left[\begin{array}{lll}0 & 0 & 1\end{array}\right]^{\top}$ and $r_{g}^{i}$ is the center of mass of link $i$ expressed in frame $\Psi_{i}$.

\section{Ship Motion Prediction}

Due to the stochastic nature of the forces that act on ships, ship motion prediction is a very difficult problem. In this section we present two simple and computationally efficient methods for predicting the future motion of the ship: the Auto-Regressive (AR) predictor and a predictor using a superposition of sinusoidal waves representation. The Auto-Regressive (AR) predictor is an all-pole model (i.e., no inputs) and gives us a model of the output directly without the need for information about the forces that cause the motion. We write

$$
y(t)=-a_{1} y(t-1)-a_{2} y(t-2)-\cdots-a_{n} y(t-n) .
$$

and define

$$
\begin{aligned}
\phi(t-1) & =\left[\begin{array}{llll}
-y(t-1) & -y(t-2) & \ldots & -y(t-n)
\end{array}\right]^{\top} \\
\theta & =\left[\begin{array}{llll}
a_{1} & a_{2} & \ldots & a_{n}
\end{array}\right]^{\top}
\end{aligned}
$$

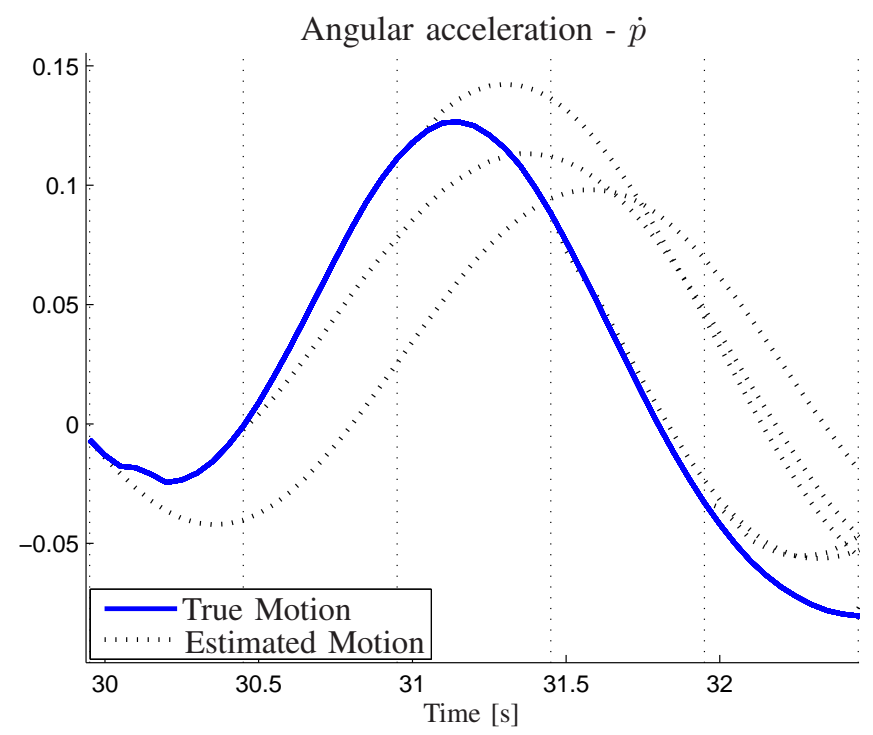

Fig. 3. A typical example of true and predicted motion (AR) where a new prediction is done every $0.5 \mathrm{~s}$. This clearly shows the need for recomputation of the predicted motion at short time intervals. In this case, the angular acceleration around the $x$-axis (roll) is shown.

Collecting $N$ samples and stacking $\phi$ in $\Phi$ and $y$ in $Y$ we find the optimal parameters $\theta$ in the least squares sense by

$$
\theta=\left(\Phi^{\top} \Phi\right)^{-1} \Phi^{\top} Y
$$

Alternatively we can fit the superposition of several sines to the measurements in the least squares sense. Following the approach in [16] we write

$$
\xi(t)=\sum_{i=1}^{N} A_{i} \sin \left(\omega_{i} t+b_{i}\right)
$$

where $A_{i}$ is the amplitude of the sines, $\omega_{i}$ is the frequency and $b_{i}$ is the phase. Assuming the frequencies are found from the peaks in the frequency spectrum, the problem amounts to finding $A_{i}$ and $b_{i}$. We can write

$$
\xi(t)=\sum_{i=1}^{N} a_{2 i-1} \sin \left(\omega_{i} t\right)+a_{2 i} \cos \left(\omega_{i} t\right)
$$

where $a_{2 i-1}=A_{i} \cos \left(b_{i}\right)$ and $a_{2 i}=A_{i} \sin \left(b_{i}\right)$ to handle phase shifts. The parameters

$$
\theta=\left[\begin{array}{lll}
a_{1} & a_{2} & \ldots \\
a_{2 N}
\end{array}\right]^{\top}
$$

representing the best fit in the least squares sense is then found from (9) with

$\phi(t)=\left[\begin{array}{lllll}\sin \left(\omega_{1} t\right) & \cos \left(\omega_{1} t\right) & \ldots & \sin \left(\omega_{N} t\right) & \cos \left(\omega_{N} t\right)\end{array}\right]^{\top}$

\section{A. Empirical Data}

The need for empirical data is of utmost importance when verifying the performance of prediction algorithms. Most publications on the topic of ship motion prediction use computer generated data such as a combination of sines, a wave model or a sine with added noise. This will not result in a good performance indicator because of the stochastic 
nature of the waves. In this work we use measurements from a real full-scale ship. This lets us compare the different prediction algorithms on real data, and most importantly gives us valuable information about the accuracy of the prediction for different prediction horizons. The ship used to collect the measurements was the RS 113 "Erik Bye" which is a 20.4 meter long Emmy Dyvi class ship and weighs 96 tons. The wave height during the experiments was about 1 meter. The ship is owned by Redningsselskapet AS, Norway.

The most important information when including the future motion of the ship in a model predictive control approach is to have as accurate predictions as possible of the velocity and the acceleration entries of the state. If gravity plays an important role, the orientation of the ship should also be included. Figure 3 shows the true and predicted angular acceleration (roll) of the ship. We see that the predicted acceleration needs to be re-computed at short time intervals to maintain a low prediction error. In general we get very good results when the predictions are computed every 0.5 or 1.0 seconds. For predictions up to 3 seconds the predictions are also reasonable and no large errors occur. If the prediction horizon is longer than 3-5 seconds we find that in some cases the predictions are out of phase which leads to large errors.

To get a more structured formulation of how the error changes with the prediction horizon we look at how the standard deviation evolves over time. This is important as it allows us to include uncertainty in the cost function and minimize this. Figure 4 shows the standard deviation for the six degrees of freedom of the velocity state denoted $\nu=\left[\begin{array}{llllll}u & v & w & p & q & r\end{array}\right]^{\top}$. We see that the AR predictions are more accurate than the superposition of sines, except for horizons of 0.2 seconds or less. We thus choose the AR method to predict the ship motions to be used in the motion planning and control presented in the next sections.

\section{Motion Planning And Control}

In this section we discuss the motion planning problem, i.e., to take the manipulator from an initial configuration to a desired end configuration using as little torque as possible. By planning the motion so that the inertial forces contribute to the motion, and don't work against it, we will, in addition to save energy, reduce the strain and tension on the manipulator. This allows for more accurate trajectory tracking and reduces the wear and tear on the manipulator.

\section{A. Motion Planning}

Consider the control law

$$
\tau=\tau_{f f}+\tau_{P D}
$$

where

$$
\begin{aligned}
\tau_{f f}= & \underbrace{M_{q q} \ddot{q}_{d}+\bar{C}_{q q} \dot{q}_{d}}_{\text {tracking terms }}+\underbrace{M_{q V} \dot{V}_{0 b}^{b}+\bar{C}_{q V} V_{0 b}^{b}}_{\text {compensation for inertial forces }} \\
& -\underbrace{\sum_{n=1}^{n}\left(J_{i} \operatorname{Ad}_{g_{0 i}}^{\top} F_{g}^{i}\right)}_{\text {gravity compensation }} \\
\tau_{P D}= & \underbrace{K_{P}\left(q_{d}-q\right)+K_{D}\left(\dot{q}_{d}-\dot{q}\right)}_{\text {PD-controller }}
\end{aligned}
$$
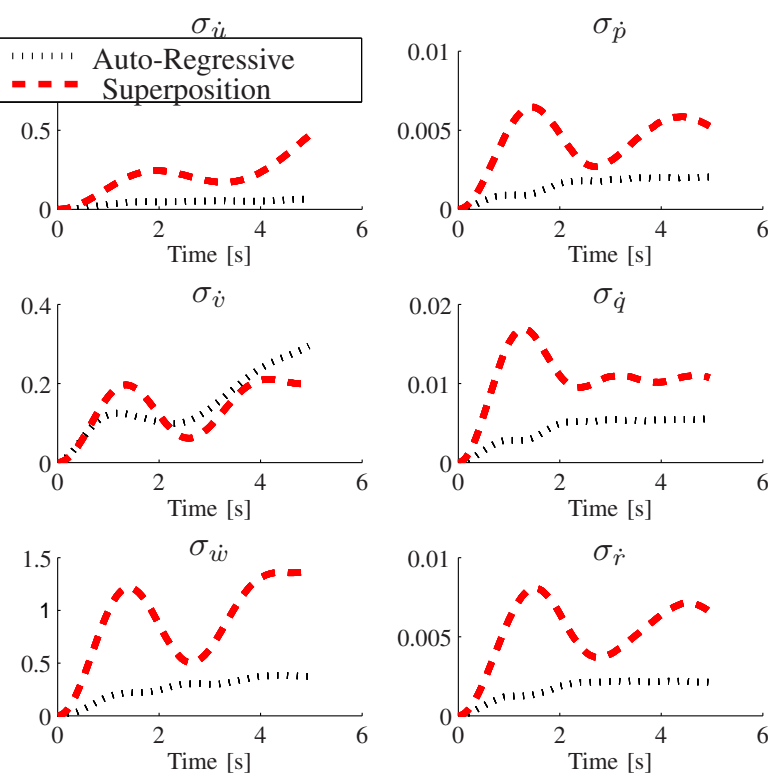

Fig. 4. The standard deviation as a function of the prediction horizon. We see that the standard deviation is smaller with the AR model, except for predictions of 0.2 seconds or less (not visible on the figure). The standard deviation is calculated from 200 samples, for "Erik Bye" moving at 15 knots at $1 \mathrm{~m}$ wave hight.

This is the standard augmented PD control law which in our case also compensates for the inertial forces. Based on the predictions of $V_{0 b}^{b}$ and $\dot{V}_{0 b}^{b}$ for a given horizon this control law tries to cancel these disturbances regardless of whether they contribute to the desired motion or not.

For trajectory tracking this is in general a very energy demanding solution. When large inertial forces are present, simply canceling these terms as in Equation (14) may require excessive joint torques. Thus, instead of regarding these terms as disturbances, we can include the prediction of the ship motion in the motion planning. The planner can then use this information to calculate the path that requires the least actuator torque for the given base motion. For example, consider a manipulator which is required to move from the left to the right on the ship. If we choose to start the trajectory at a time when the inertial forces contribute to the desired motion we can get an almost free ride from one side to the other. If we simply choose to cancel these disturbances, for example by Equation (14), we might end up following a trajectory for which the inertial forces work against the desired motion for the entire interval. One intuitive situation where this can occur is when the manipulator moves uphill instead of downhill for the entire motion and thus not taking advantage of the gravitational forces.

We follow the approach presented in From et al. [3] and solve the motion planning problem by numerically minimizing an objective function representing the joint torques

$$
P=\min _{t_{0}, t_{1}} \int_{t=T_{0}}^{T_{1}} \tau^{\top} D \tau d t
$$

where $T_{0} \leq t_{0}<t_{1} \leq T_{1}$ and $D$ is a positive definite matrix that defines a metric in $\tau$-space. In From et al. [3] this was 


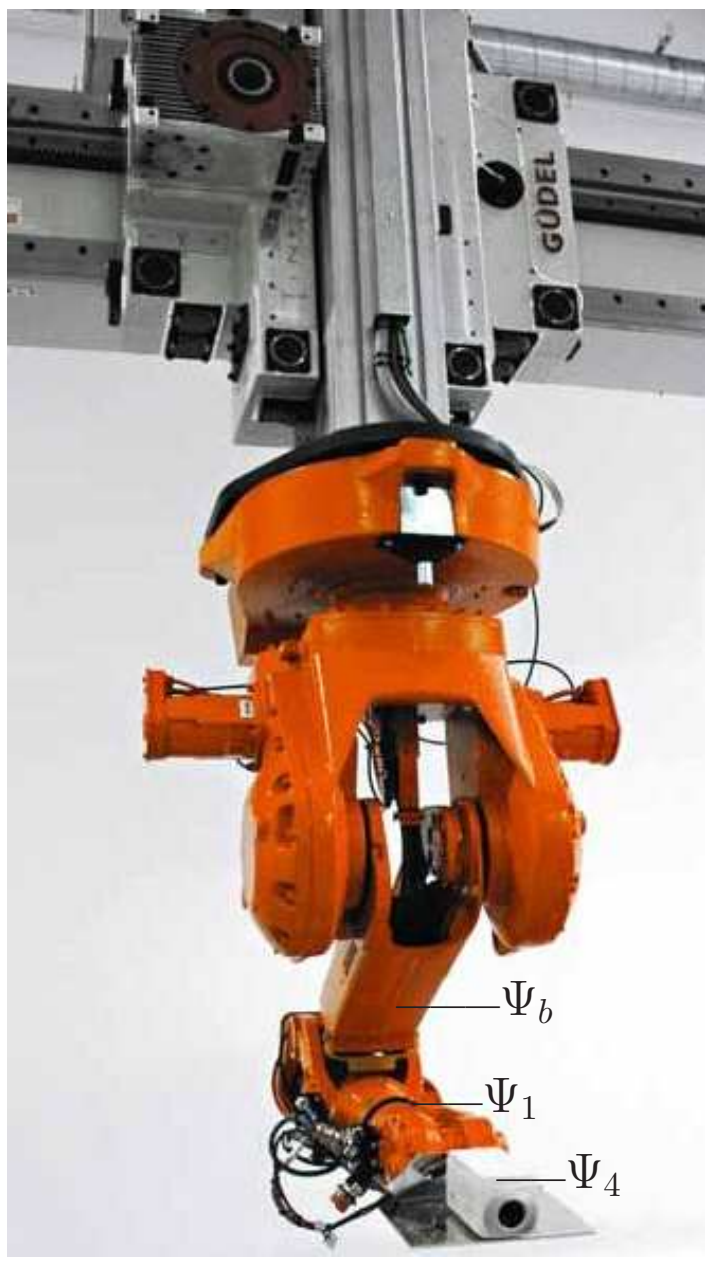

Fig. 5. ABB robot mounted on a gantry crane. The first 5 joints (the 3 of the gantry crane and the first 2 of the robot) are used to generate the ship motion and the last four joints of the robot are used for optimal motion planning of a 4-DoF robot. Links 2 and 3 of the robot are hidden in the wrist. Courtesy ABB Strategic R\&D for Oil, Gas and Petrochemicals.

solved assuming the base motion was known. We now use realistic predictions of the base motion in the motion planner. These predictions will become less accurate as the horizon increases and we investigate how the choice of prediction horizon affects the performance of the motion planner.

From Section IV we see that the accuracy of the predictions is different for the different axes. For the AR predictor we get that the predictions of the acceleration about the $y$ axis is less accurate than about the $x$ - and $z$-axes. Also, the linear acceleration in the direction of the $x$-axis is far more accurate than the $y$ - and $z$-axes.

We now present a modified cost function that minimizes also the expected variance on the output assuming we know how the variance evolves with time for different degrees of freedom, i.e., the standard deviation shown in Figure 3. In Cannon et al. [20] the control objective of the Stochastic MPC law is to regulate the expected value and variance of the output state. In the following we apply the same ideas and include the covariance matrix in the cost function so that a trajectory that also minimizes the covariance is chosen. We will use a cost function similar to the one found in Cannon et al. [20] given by

$$
P=\sum_{j=0}^{N-1} l(k+j \mid k)+L(k+N \mid k)
$$

where $L(k+N \mid k)$ is the cost-to-go and

$$
l(k+j \mid k)=\bar{q}^{2}(k+j \mid k)+\kappa\left\|\Sigma_{q}(k+j \mid k)\right\|_{r}
$$

with

$$
\bar{q}(k+j \mid k)=\mathbb{E}[q(k+j \mid k)]
$$

denoting the expected value and

$\Sigma_{q}=\left[\begin{array}{ccc}\mathbb{E}\left[\left(q_{1}-\bar{q}_{1}\right)\left(q_{1}-\bar{q}_{1}\right)\right] & \ldots & \mathbb{E}\left[\left(q_{1}-\bar{q}_{1}\right)\left(q_{n}-\bar{q}_{n}\right)\right] \\ \vdots & \ddots & \vdots \\ \mathbb{E}\left[\left(q_{n}-\bar{q}_{n}\right)\left(q_{1}-\bar{q}_{1}\right)\right] & \ldots & \mathbb{E}\left[\left(q_{n}-\bar{q}_{n}\right)\left(q_{n}-\bar{q}_{n}\right)\right]\end{array}\right]$

so that $\Sigma_{q}(k+j \mid k)$ is the covariance matrix of $q(k+j \mid k)$ given the measurements of the robot state $q(i)$ and the ship state $g_{0 b}(i)$ for $i=k_{0} \ldots k$ and $\|\cdot\|_{r}$ denotes the Euclidean norm of each row. The relative weighing of the expected value and the variance can thus be controlled directly through the parameter $\kappa$. Similarly, we add the second part of (18) to the cost in (16) and get the cost function

$$
P=\min _{k_{0}, k_{1}} \sum_{k=K_{0}}^{K_{1}}\left(\tau^{\top}(k) D \tau(k)+\kappa\left\|\Sigma_{q}\left(k \mid K_{0}\right)\right\|\right) .
$$

where $\left\|\Sigma_{q}\right\|$ denotes the Euclidean norm of the covariance matrix. The problem is thus to find the start time $k_{i, 0}$ and the end time $k_{i, 1}$ for the motion of each joint subject to the restriction $K_{0} \leq k_{i, 0}<k_{i, 1} \leq K_{1}$. The cost, however, sums over the entire pre-defined interval $\left(K_{0}, K_{1}\right)$, i.e., also when $\dot{q}_{i}(k)=0$. We note that in this case we do not necessarily have $\tau_{i}(k)=0$ because of the inertial forces.

We will assume that each degree of freedom of the ship motion has a normal distribution, i.e.,

$$
\left[\begin{array}{c}
\dot{u} \\
\dot{v} \\
\vdots \\
\dot{r}
\end{array}\right] \sim\left[\begin{array}{c}
\mathcal{N}\left(\overline{\dot{u}}, \sigma_{\dot{u}}^{2}\right) \\
\mathcal{N}\left(\dot{\bar{v}}, \sigma_{\dot{v}}^{2}\right) \\
\vdots \\
\mathcal{N}\left(\dot{\dot{r}}, \sigma_{\dot{r}}^{2}\right)
\end{array}\right]
$$

where $\sigma_{x}^{2}=\mathbb{E}\left[(x-\bar{x})^{2}\right]$ is the variance of $x$. Examples of the expected value and the variance for the acceleration of the ship are shown in Figures 3 and 4, respectively. Similar relations can also be found for the position and velocity of the ship.

For linear systems the Kalman filter gives us the expected state and the error covariance. For non-linear systems we use the Extended Kalman filter i.e., we linearize around the mean value, and find the expected state of the robot and the covariance matrix used in (18). This allows us to include both the expected state and the covariance at time $(k+j)$ given the measurements available at time $k$ also for nonlinear systems. This is then included in the cost function (18) and the optimal solution is found by minimizing the weighed cost of the expected value and the covariance. 

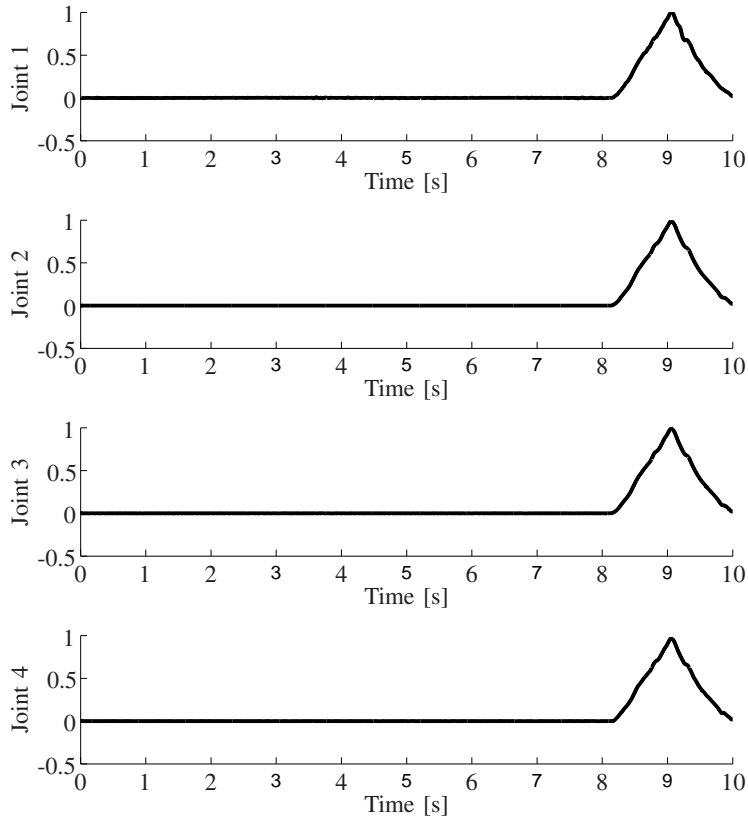

(a) Joint Velocities.
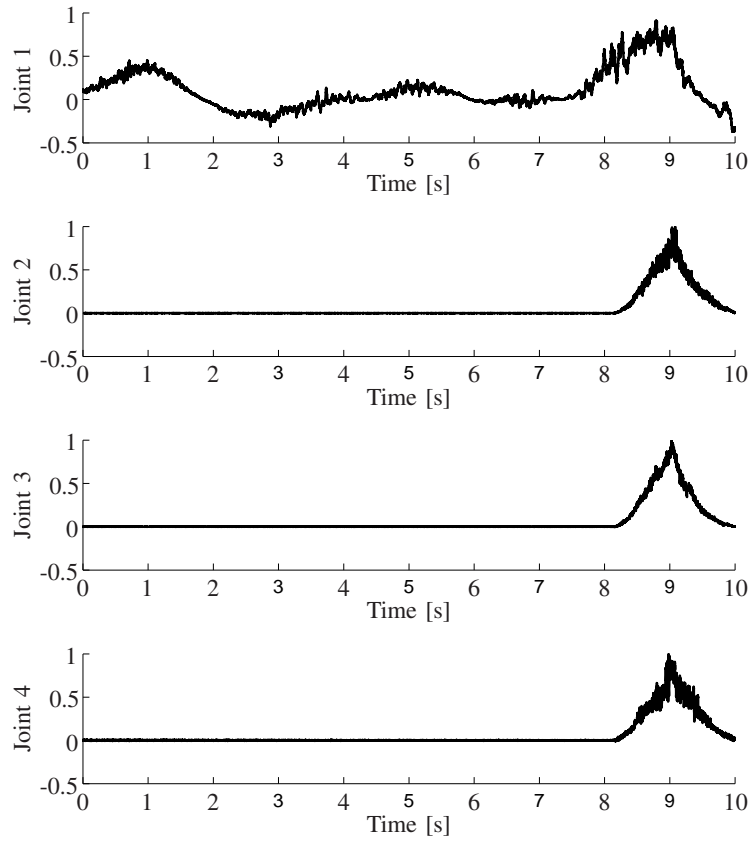

(b) Torques times velocities.

Fig. 6. The joint velocities and the torques times velocity for a simple trajectory are shown for all joints. Note the mismatch between the torques and the velocities for joint 1 . This is due to the large amount of torque needed to compensate for the inertial motion.

\section{EMPIRICAL STUDiES}

In this section we present the simulation results and the experimental results from the lab. Due to the stochastic nature of the disturbances, simulations are important to be able to perform a sufficient number of runs and get statistically meaningful results. We also run some of the simulations on a ship-manipulator system in the lab to verify the results and the computational feasibility of the approach.

\section{A. Simulation and Experimental Setup}

Both the simulations and experiments were performed using true motion data from the full scale ship "Erik Bye" and predictions from the AR model as presented in Section IV. All the data sets are picked from one long sampling and are thus collected during a short period of time and in a sea state for which the dominant components of the motion were in the xz-plane, i.e., the pitch is far bigger than the roll, and the sway is almost zero. In other words, all the data sets are measurements of the ship moving with the same velocity, in the same sea state and with the same attack angle on the waves.

For the experimental setup we used a 9-DoF robot (3-DoF gantry crane and 6-DoF industrial manipulator, see Figure 5) where we used the first 5-DoF to generate the ship motion. This allowed us to generate the surge, sway and heave motion with the gantry crane and the roll and pitch motion with the first two joints of the manipulator. The yaw motion is very small and can be neglected. The last four links of the manipulator can then be considered a standard manipulator for which we were to choose the optimal control and motion planning. As the motion of the "base", i.e., link 2 of the manipulator, was set to exactly the same as the measurements taken from the full scale ship this setup allows us to perform very realistic experiments.

\section{B. Motivation}

We start by running an experiment where we let all the joints follow a simple trajectory in joint space when the base moves exactly like the motion of the ship. The trajectory chosen takes each joint from the initial position $q_{i}(8)=0$ to a target position $q_{i}(10)=\frac{\pi}{2}$ in 2 seconds. The motion starts after 8 seconds so the joint torques of each joint when the robot does not move (but the base does) is also obtained. In each joint there is a high-gain independent joint controller to guarantee that the desired trajectory is followed.

The first link is very heavy, while the last three are lighter links (about $10 \%$ of the weight of link 1). Figure 6(a) shows the velocities and Figure 6(b) shows torque times velocity for this simple trajectory. Note that for link 1 more torque is used to compensate for the inertial forces than to follow the desired trajectory. We see that the torque required to compensate for the inertial forces, even when the joint does not move, is substantial. This is due to the large inertia of link 1, compared to links 2, 3 and 4 . This experiment clearly illustrates that for joints that need to move links with large inertia, the inertial forces due to the moving base need to be compensated for and should be taken into consideration in the motion planning of the manipulator. 

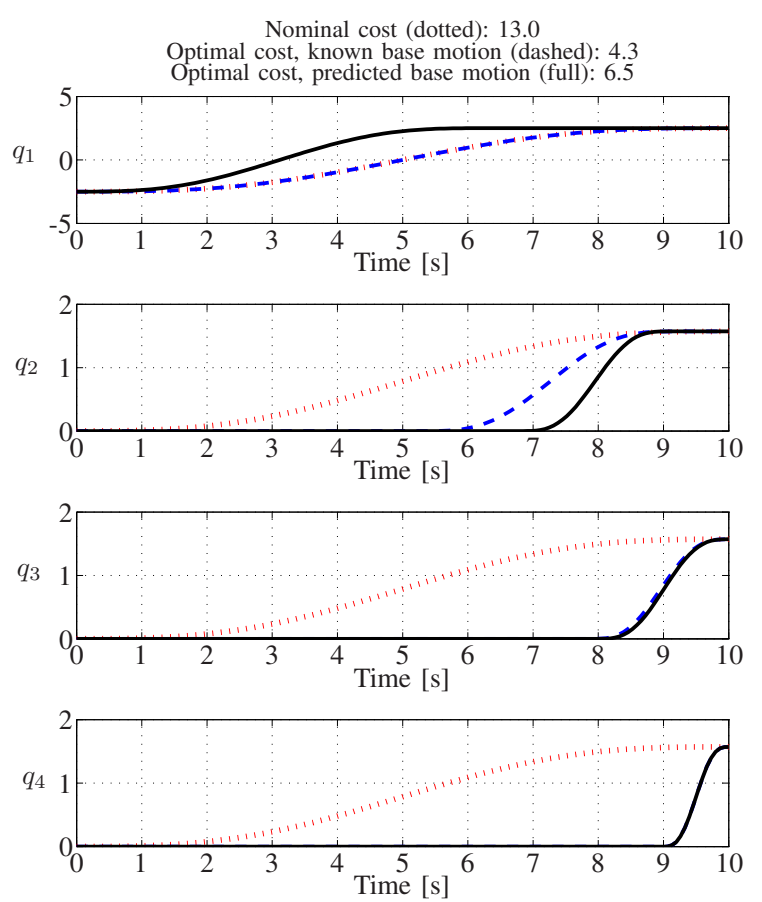

(a) Torque-based cost function.
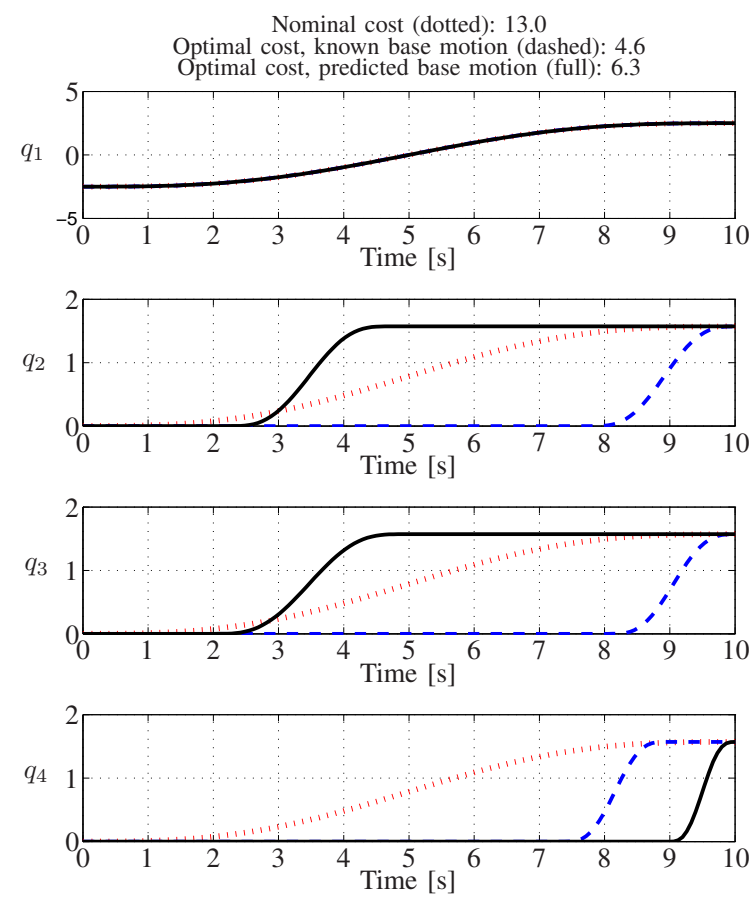

(b) Torque and variance-based cost function.

Fig. 7. Optimal motion trajectory for a 4-DoF manipulator from the experimental setup. Three different trajectories are shown: a baseline trajectory with maximum motion duration (dotted, red line), an optimized trajectory taking the correct base motion into account (dashed, blue lines), and an and an optimized trajectory taking the predicted base motion into account (full, black lines).

\section{Motion Planning in the Presence of Uncertainties}

In this section we show how we can exploit the inertial forces and choose a trajectory that minimizes the wear and tear on the manipulator. We follow the approach presented in From et al. [3] and solve the motion planning problem by numerically minimizing an objective function (16). We let $D$ be a positive definite matrix that defines a metric in $\tau$-space. For a given interval $\left(T_{0}, T_{1}\right)=(0,10)$, the optimization problem is then to find the start and the end time for each joint with the restriction that $T_{0} \leq t_{i, 0}<t_{i, 1} \leq T_{1}$ for all $i$. The shape, but not the starting time or length, of the trajectory is thus assumed fixed. By fixing the shape, we find a sub-optimal solution that can be solved efficiently and in real time. Finding the optimal solution over all trajectories is a huge optimization problem and computationally too demanding to be solved in real time. The start and end configurations are chosen as

$$
\begin{aligned}
& q\left(T_{0}\right)=\left[\begin{array}{llll}
0 & 0 & 0 & 0
\end{array}\right]^{\top}, \\
& q\left(T_{1}\right)=\left[\begin{array}{llll}
\frac{\pi}{2} & \frac{\pi}{2} & \frac{\pi}{2} & \frac{\pi}{2}
\end{array}\right]^{\top} .
\end{aligned}
$$

The motion planning problem thus amounts to finding the eight parameters (one start and end time for each joint) that minimize the total squared required torque integrated over a fixed time interval while starting and ending the motion in the required configurations. We choose $D=$ $10^{-6} \cdot \operatorname{diag}\left[\begin{array}{llll}10 & 2 & 2 & 5\end{array}\right]$ reflecting the masses of the joints.

We also compute the optimal trajectory minimizing the cost function (21) which also includes the covariance matrix.
We choose $\kappa=10$ to enhance the effects of adding the covariance matrix and let $\|\cdot\|$ denote Euclidean norm (spectral norm) of the covariant matrix.

We now discuss how to choose the horizon and the frequency at which the problem is re-solved in a receding horizon setting when only predictions of the base motions are known. In the previous section we found that the predictions are very accurate for about 0.5 seconds and relatively accurate for a horizon of about 3 seconds. When it comes to motion planning, however, we find that a longer horizon should be chosen. The reason for this is that we search for the time interval for which the inertial forces contribute to the motion as much as possible. We thus want to choose the horizon length as long as possible, but at the same time avoid using predictions that are not accurate and may lead to large errors.

First we run several simulations to guarantee statistically meaningful results using the two cost functions described. Table I shows the optimal horizon, the total cost and the maximum torques for i) a nominal trajectory which starts at $t_{i, 0}=T_{0}$ and ends at $t_{i, 1}=T_{1}$ for all joints, ii) the cost function (16) minimizing the torques only, and iii) the cost function (21) minimizing the torques and variance. In Table I we show the average values of 200 simulations and we show the values of the cost function based on torque only for both approaches in order to be able to compare the two values.

We note that the optimal horizon increases from 4 to 6 seconds when the variance is included in the cost function. 
We also note that the cost decreases somewhat and, more importantly, the maximum torque decreases substantially. This is a great advantage and will reduce the wear and tear on the manipulator.

Finally we let a real robot in the lab follow the same trajectory. This empirical study is important in order to verify the simulation results. Figure 7 shows one example of the optimal trajectories for the two cost functions for the experimental set-up. Both trajectories are calculated in real time every 2 seconds with horizon lengths of 4 and 6 seconds, respectively. We note that adding the covariance to the cost function results in a completely different trajectory.

We do the empirical studies with 5 different base motions. The base motions for both the simulations and the experiments are taken from the same data set so the results can be compared directly. We find that the value of the cost function is about the same for the two cost functions. Similarly to the simulations, we find that the value of the maximum torque is reduced with about $30 \%$. The experimental results thus support the tendencies found in the simulations and we conclude that adding the covariance to the cost function has a positive effect on the maximum torque.

Most importantly we see that adding information about the predicted state of the ship_-even though these predictions are accurate only to a certain extent - can substantially reduce the torque needed to follow the given trajectory. Reducing both the energy used and the maximum torques with about $40-60 \%$ will drastically reduce the strain and tension on the manipulator, which is what we wanted in the first place.

\begin{tabular}{l|ccc} 
Cost function & Horizon [s] & Cost & Maximum Torque [Nm] \\
\hline Nominal & & 10.8 & 565 \\
Torque & 4 & 6.3 & 343 \\
Torque and variance & 6 & 6.1 & 230
\end{tabular}

TABLE I

OPTIMAL HORIZON AND THE CORRESPONDING COST AND MAXIMUM TORQUES FOR THE TWO COST FUNCTIONS BASED ON 200 SIMULATIONS.

\section{CONCLUSIONS}

In this paper we study the effects that ship motion prediction accuracy have on the motion planning and control of robots mounted on ships. The main difference from conventional motion planning is that large inertial forces enter the robot dynamics due to the motion of the base. This paper presents the first detailed study of how these inertial forces affect the dynamics of the robot and we find that they do so to such an extent that they cannot be ignored. We have put great effort in making the experiments as realistic as possible. To this end we have collected ship motion data from a full scale ship and performed several experiments with a robot mounted on a forced base. These experiments show that if the predicted inertial forces are included in the motion planner in a receding horizon setting, we can utilize these forces to more cheaply move from an initial to a target configuration.

\section{ACKNOWLEDGMENTS}

P. From and T. Gravdahl wish to acknowledge the support of the Norwegian Research Council and the TAIL IO project for their continued funding and support for this research. The TAIL IO project is an international cooperative research project led by Statoil and an R\&D consortium consisting of ABB, IBM, Aker Solutions and SKF. While working on this paper the first author was visiting the University of California at Berkeley.

\section{REFERENCES}

[1] L. J. Love, J. F. Jansen, and F. G. Pin, "On the modeling of robots operating on ships," IEEE International Conference on Robotics and Automation, Louisiana, USA, vol. 3, pp. 2436-2443, 2004.

[2] J. Kitarovic, V. Tomas, and D. Cisic, "The electronic and informatics age - a new stage in developing highly effective ships," 47th International ELMAR Symposium, Zadar, Croatia, pp. 385-388, 2005.

[3] P. J. From, V. Duindam, J. T. Gravdahl, and S. Sastry, "Modeling and motion planning for mechanisms on a non-inertial base," International Conference of Robotics and Automation, Kobe, Japan, 2009.

[4] S.-R. Oh, K. Mankala, S. Agrawal, and J. Albus, "Dynamic modeling and robust controller design of a two-stage parallel cable robot," IEEE International Conference on Robotics and Automation, Louisiana, USA, pp. 3678-3683, 2004.

[5] D. C. Salzmann, "Ampelmann prototype - developing a motion compensating platform for offshore access," European Wind Energy Conference, Milan, Italy, 2007.

[6] G. Lebans, K. Wilkie, R. Dubay, D. Crabtree, and T. Edmonds, "Telerobotic shipboard handling system," OCEANS, Nova Scotia, Canada, vol. 2, pp. 1237-1241, 1997.

[7] K. Kosuge, M. Okuda, and T. Fukuda, "Motion control of manipulator/vehicle system floating on water," Proc. of 2nd IEEE International Workshop on Advanced Motion Control, Nagoya, Japan, pp. 12611268, 1992.

[8] H. Kajita and K. Kosuge, "Force control of robot floating on the water utilizing vehicle restoring force," IEEE/RSJ International Conference on Intelligent Robot and Systems, Grenoble, France, vol. 1, pp. 162 167, 1997.

[9] T. Yoshikawa, K. Harada, and A. Matsumoto, "Hybrid position/force control of flexible-macro/rigid-micro manipulator systems," IEEE Transactions on Robotics and Automation, vol. 12, no. 4, pp. 633 640, 1996.

[10] A. Bowling and O. Khatib, "Design of macro/mini manipulators for optimal dynamic performance," Proc. IEEE International Conference on Robotics and Automation, New Mexico, USA, vol. 1, pp. 449-454, 1997.

[11] S. McMillan, D. E. Orin, and R. B. McGhee, "Efficient dynamic simulation of an underwater vehicle with a robotic manipulator," IEEE Transactions on systems, man and cybernetics, vol. 25, no. 8, pp $1194-1206,1995$.

[12] O. Egeland and J. R. Sagli, "Coordination of motion in a spacecraft/manipulator system," International Journal of Robotics Research, vol. 12 no. 4, pp. 366-379, 1993.

[13] X. Yang, H. Pota, M. Garratt, and V. Ugrinovskii, "Ship motion prediction for maritime flight operations," Proc. World Congress IFAC, 2008

[14] — - "Prediction of vertical motions for landing operations of uavs," Proc. IEEE Conference on Decision and Control, 2008.

[15] A. Khan, C. Bil, and K. E. Marion, "Ship motion prediction for launch and recovery of air vehicles," Proceedings of MTS/IEEE OCEANS, 2005.

[16] J. C. Chung, Z. Bien, and Y. S. Kim, "A note on ship-motion prediction based on wave-excitation input estimation," IEEE Journal of oceanic engineering, vol. 15, no. 3, 1990.

[17] V. Duindam and S. Stramigioli, "Lagrangian dynamics of open multibody systems with generalized holonomic and nonholonomic joints," in Proceedings of the IEEE/RSJ International Conference on Intelligent Robots and Systems, California, USA, October 2007, pp. 3342-3347.

[18] _ - "Singularity-free dynamic equations of open-chain mechanisms with general holonomic and nonholonomic joints," IEEE Transactions on Robotics, vol. 24, no. 3, pp. 517-526, June 2008.

[19] R. M. Murray, Z. Li, and S. S. Sastry, A Mathematical Introduction to Robotic Manipulation. CRC Press, 1994.

[20] M. Cannon, P. Couchman, and B. Kouvaritakis, "Mpc for stochastic systems," Assessment and Future Directions of Nonlinear Model Predictive Control, vol. 358, 2007. 